\title{
3D scanning data use for modular building renovation based on BIM model
}

\author{
Anatolijs Borodinecs ${ }^{1, *}$, Jurgis Zemitis ${ }^{1}$, Modris Dobelis ${ }^{2}$ and Maris Kalinka ${ }^{3}$ \\ ${ }^{1}$ Institute of Heat, Gas and Water Technology, Faculty of Civil Engineering of Riga Technical \\ University, Riga, Latvia \\ ${ }^{2}$ Department of Computer Aided Engineering Graphics, Faculty of Civil Engineering of Riga \\ Technical University, Riga, Latvia \\ ${ }^{3}$ Department of Geomatics, Faculty of Civil Engineering of Riga Technical University, Riga, Latvia
}

\begin{abstract}
Nowadays new and modern tools should be actively used in buildings energy retrofitting processes. Handmade measuring and 2D design process should be minimized as possible and by replaced by advanced computer technologies such as automated data proceedings, 3D scanning and automated production lines. All these measures will allow significant minimization failures in design, energy simulation and construction. Available computing capacities allows data proceeding in reasonable time and quality. 3D building model is an efficient tool to develop precise retrofitting project and to make correct energy consumption estimation3D building model allows precise dynamic energy simulation using such software as IDA-ICE, RISUKA, IES, EnergyPlus etc. This research provides detailed analysis of existing buildings whole 3Ddevelopment process from scanning to creation of model.
\end{abstract}

\section{Introduction}

3D laser scanning techniques have been developed since the end of 1990s for 3D digital measurement, documentation and visualization in many fields including geospatial and survey industries, architecture, processing and mining industries, building infrastructure, archaeology, and etc. [2-3]. Latest advances in hardware and increased BIM software performance are helping to overpass to a new lev-el of scanning application for the building construction industry. The scanning of objects most often is applied to the existing building structures. The leading scanner manufacturers claim that scanning technology is becoming a critical function necessary to complete the integrated BIM cycle efficiently and provides a clear value-add for the integrated BIM work-flow [4].

BIM is a digital data flow surrounding the lifecycle of an asset or element of the built environment, intended to provide better information management to aid with decision making. As a process, BIM has been gaining global acceptance across the Architecture, Engineering, Construction, and Operations (AECO) community for improving information

\footnotetext{
* Corresponding author: anatolijs.borodinecs@ rtu.lv
} 
sharing about built assets. A key component of this is an object-based 3D parametric model that holds both geometric and semantic information. Other related BIM tools can be utilized to ex-tract useful information for various purposes [5].

The captured 3D building model serves two purposes. One is for energy analysis before and after reconstruction when the selected type and/or variant of insulation solution is applied. The use of BIM concept in building industry allows fast and easy assessments of several potential solutions considered within the same BIM model to choose the most advantageous.

The second goal is an elaboration of a de-tailed structural design for the selected solution, which can be later streamlined from CAD software directly to CNC fabrication site. Many project subcontractors are very sophisticated in their ability to create physical work assemblies in off-site locations and then bring them on-site in large clusters for rapid installation. Prefabrication offers many benefits, including safer working conditions, controlled environments, and automated machine usage [21]. However, prefabrication can only be successful when used in conjunction with accurate information about the destination of the final installation, which laser scanning can provide much faster [4].

\section{Creating as-built models}

\subsection{A Subsection Sample}

A growing number of building renovations concerning energy saving demands the acceleration or industrialization of this process which in turn requires a presence of three key elements [6-7]:

$\checkmark$ A technical concept allowing insulation by the assembly of standardized modular components with a minimum of specific components;

$\checkmark$ A chain of stakeholders including renovation owner, renovation designers, architects, modular component suppliers, and installation teams;

$\checkmark$ A digital engineering chain that can pro-vide aiding tool for supporting each task of the renovation process.

These processes are highly collaborative and all the stakeholders need to share data, information and knowledge at the various steps of the renovation process. Most of the times they will want to use their own specific soft-ware tool (scanning, CAD, finite element analysis, heating simulation, energy analysis, lighting simulation, cost estimators, computer aided manufacturing, transport optimization, project manager etc.). In order to guaranty a minimum of consistency, it is necessary to share the same renovation model between all the stakeholders and therefore to support the whole process with a BIM compatible software [8].

Recent advances of IT use in renovation projects have proved that BIM is mostly accepted by specialists consuming different tools in industry [9]. BIM model is used to perform expert services for buildings such as energy analyses. Therefore, two types of expert soft-ware might interact with a BIM model: (1) da-ta input applications providing services of import, data capture and monitoring, data processing or transformation of captured data into BIM or (2) data output applications providing reports or technical analyses such as energy analyses, structural analyses or clash detections [10].

\subsection{Advantages of BIM use in renovation}

Effective use of BIM can be achieved if it is implemented from the initial stage of a project. BIM applied effectively considerably saves the time in the design phase compared to the 
traditional approach. One of the potential advantages of BIM applications in renovation is the ability to assess the energy performance by creating model and investigating several alternatives of energy simulations numerically before the optimal solution is approved. Therefore implementation of BIM workflow and providing early energy simulation could increase efficiency of the whole renovation process.

2D models in the form of drawings are not capable of handling complicate design and management of large, complex construction projects, as well as information flow throughout the entire process of the project. BIM models instead, represent elements as semantic objects rather than simple spatial elements. For example, walls in the model are separate, volumetric objects with multiple surfaces which define relationships between adjacent building elements (walls, doors, windows, slabs, roof, outside environment, soil, etc.) and retain the relevant physical of properties material.

One of the main benefits of BIM is the ability to create an effective early collaboration between project participants. In order to avoid data conflicts and unnecessary reworks, there must be an appropriate management system. Furthermore, early involvement of contractors in the design phase makes it possible to take more straight decisions on cost estimation and arranging the construction materials. However, there are and always will be several restrictions due to data transfers between different software tools and legal contract issues [10].

\subsection{Implementing BIM}

BIM implementation for an old building has other challenges and capabilities compared to the new construction which already originally was designed using contemporary technologies. No documentation is available in the required BIM format in former case. BIM adoption in practice for the buildings designed and built more than ten years ago is rather expensive and complicate process. The creation of an as-built BIM involves measuring the geometry and appearance of an existing facility and transforming those measurements into a high-level, semantically rich 3D model representation.

The creation of as-built BIM model mainly involves three tasks [11]: modelling the geometry of the building elements, assigning an object category and material properties to elements, and establishing relationships between elements. The task of geometric modeling is to capture a representation of the building elements by fitting 3D geometric primitives to the point cloud data [11]. Geometric primitives have to be individual volumetric shapes, e.g. a simple wall can be modeled as a rectangular box (cuboid). More complex structures are not required in the present study, but they may be modeled non-parametrically, e.g. using triangular meshes. Since BIMs are normally defined as solid shapes, surface-based representations if present need to be transformed into solid models of corresponding building elements.

The capturing of parametric elements in external modelling applications depends upon the outcome of desired scope. Depending on the degree of fidelity [12] of the model several levels may be distinguished. In a low-fidelity model rooms or spaces are modelled as simple solid volume geometric masses. Medium fidelity model is made up from internal walls, floors, ceilings, and doors, etc. High-fidelity model besides the former includes detailed door and window openings, detailed thermal bridge elements, MEP and etc. building elements. Within each room or space permanent fixtures may be represented, such as: sinks, toilets, urinals, lighting fixtures, etc.

In [7] the term Level Of Details (LOD) is used to characterize how much detail is included in the model element. American Institute of Architects defines Level Of Development (LOD) [13] to characterize the degree to which the element's geometry and attached information has been thought through - the degree to which project team members 
may rely on the information when using the model.

For detailed scopes of energy evaluation very specific external applications (IDA ICE, TRACE 700, IES VE, DesignBuilder, AECOsim, TRNSYS, etc.) are often used. The use of external analysis applications require a methodical approach regarding the creation of BIM model with consistent elements.

Less detailed simulation scopes can be quickly checked using very simple, user friendly and cheap solutions like Trimble SketchUp in connection with EnergyPlus and OpenStudio modules. SketchUp allows very rapid creation of both the low-fidelity and medium-fidelity models. The program supports different CAD formats, allowing the import of $2 \mathrm{D}$ drawings to be used as an underlay when creating 3D geometry. SketchUp supports OpenStudio cross-platform collection of software tools which support the whole building energy modelling process using EnergyPlus and advanced daylight analysis using Radiance.

\subsection{Modelling the geometry of the building}

The forming of as-built model is a process of creation of objects that represent building elements, including both geometric and non-geometric attributes and relationships. If BIM is modelled on the basis of previously captured building information, the preceding data capture, processing and recognition methods influence data quality through the deployed technique and the provided level of detail [9].

This was complicate process in the earlier period of the development of these technologies, but now numerous plug-in or add-on software modules have been developed so advanced that it is possible to import and process large scanning data file directly into the end user software. In addition, many hardware producers now are trying to attract more customers, allowing their proprietary data formats convert into a neutral or not-protected file format, e.g. ASCII format, in order to enable post-processing in many more different platform independent software applications.

To choose an appropriate data export format one should consider which CAD software will be used in further workflow. Most of the popular CAD and BIM software packages, e.g. Autodesk AutoCAD, Autodesk Revit, Graphisoft ArchiCAD, and Bentley AECOsim Building Designer, allow direct import from scanners like Faro, Trimble or Leica using different data formats. These formats include xyz coordinates of the points, RGB colour, and point intensity. The only challenge is to follow the never ending software version upgrades which for CAD software traditionally occur annually. When considering the application of special add-on or plug-in products from third party developers it is very important to analyse the compatibility issues. Sometimes the particular well documented module may not even work with a newer or older version of end user software it was originally developed for.

Some registration software, such as Trimble RealWorks, has the capability to create content from within the point cloud by running special algorithms across the data points and recognizing surfaces directly from it [4]. Creating objects within the registration software offers the benefit of rapid creation but has some limitations surrounding the accuracy and metadata acceptance of modelled objects. Creation of object models using external authoring applications is slower with more manual work involved but has the benefit of detailed object representation and increased metadata acceptance.

In practice, as-built BIM modelling very often is done interactively in a time consuming and error-prone process $[8,11,14]$, e.g. with the BIM compatible software from major vendors like Autodesk Revit, Bentley AECOsim, Graphisoft ArchiCAD, Trimble Tekla Structures or Nemetschek Allplan. The latest versions of this software provide powerful features for reverse engineering tasks and processing of captured point clouds. Although some allow the rapid generation of building floor plans or offer BIM integration, the 
depicted software solutions are far away from automated or semi-automated BIM modelling of existing buildings [9].

\section{Case study}

A typical two-story building of approximately 300 square meters was selected in a pilot study. This was a residential house with four apartments which was designed and built in early 1960s when there were no regulations regarding energy saving policy at all. A practical reconstruction of a BIM compatible 3D building model from 3D laser scanner data included several steps.

There are several tools for building capture such as tachometry, 2D/3D geometrical drawings, and laser scanning. These tools require strong skilled personnel in order to model the existing buildings. First step is a recapture of building information with reverse engineering processes in terms of "points to BIM" or "scan to BIM" [9].

\subsection{Laser scanning and data pre-processing}

The building is located in a suburb territory of a small town and there were no concerns about how to eliminate pedestrian "noise" from the scan data. The building plot is single rectangle with rather simple facades having no tiny details. Therefore the number of scan station locations was basically determined only by the amount of adjacent greenery and interfering objects like cables, and etc. The laser scanning of the building was performed from nine positions (Figure 1) with phase shift technology scanner FARO 3D 120. The scanning work was carried out during on-site visit on a well-lit day without precipitations.

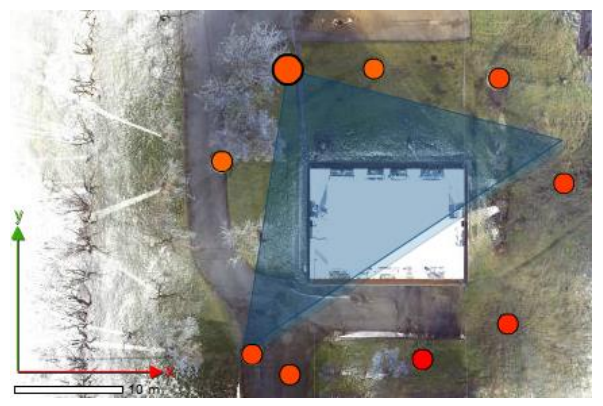

Fig. 1. Scanner positions for two-story residential house

Altogether 18 registration marks or spherical reference targets were used. The average distances between scan points on the walls were $5 \mathrm{~mm}$. The scanning time in each scanner position took about 7 minutes. The actual time spent on-site for the equipment setup, calibration and data acquisition from the arrival until the departure in this study was about 6 hours. Information about geo-referencing (orientation, location and elevation) of the site along with the neighborhood characteristic (soil type, trees, nearby buildings, etc.) required for further energy analyses was documented during the same day visit. All the procedures were performed by two persons.

Some of the metadata may be stored directly in the point cloud file format, or may be linked to the point cloud for later use in BIM models. The existing plan views and sections for the building were collected in an analogue format (blueprints) from the available inventory documentation and then digitalized. 
The data sets with about 400 million points from all scanning positions were downloaded from the scanner. Pre-processed raw scan data as point clouds were positioned and oriented in their own coordinate systems. Merging or stitching together all the point clouds in a single coordinate system was performed by Faro Scene software on a network.

Not all the scan points in the data set may be used because of some always existing noise accidentally captured. The noise points need to be filtered out or cleaned from the raw data.

For data cleaning and deleting of duplicates Faro Scene and Leica Cyclone software were used. This process took about 4 hours and included the following steps:

- $\quad$ Deleting outliers;

- Unifying the scan points to delete double points, unify the space between the points;

- Cleaning off the noise of neighboring objects like trees, bushes, cables, etc.

All these adjustments in a post processing or registration stage resulted in $3 \mathrm{~mm}$ accuracy with respect to the station. It took another 4 hours to perform the following procedures:

- Checking the convergence between the scan positions;

- Checking the conformity of the reference dimensions for e.g. windows, doors or corners with different survey methods (total station, tape measure or laser distance measuring tool). In the present study the checked dimensions are between 5-10 mm.

Since various raw data formats are used for scanners, sometimes it is necessary to convert the scanning data into another format, depending upon which modelling software is used for the post-processing. In this study *.pts and *.e57 scan data formats were required.

Raw data obtained by laser scanning are referred as point cloud, which is a set of vertices in a 3D coordinate system. These vertices define or digitally represent xyz coordinates of the points of the external surface of the building. For most of the phase-based scanners, the raw scanning data are combined as both point and intensity, so the corresponding intensity image in both 2D and 3D can be obtained, which is useful for more detailed documentation and identification of objects. Data are stored in *.pts or *.e57 formats and examples of this representation are shown in Figure 2 and Figure 3.

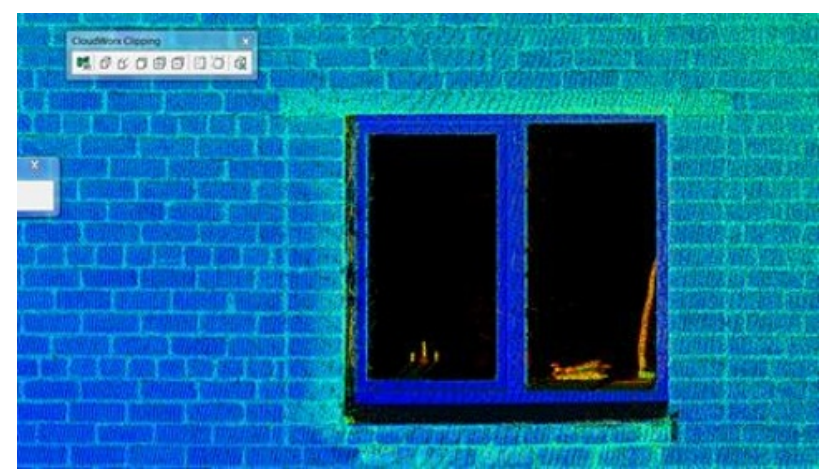

Fig. 2. Details of the building elements in point cloud visualization mode in *.pts format 


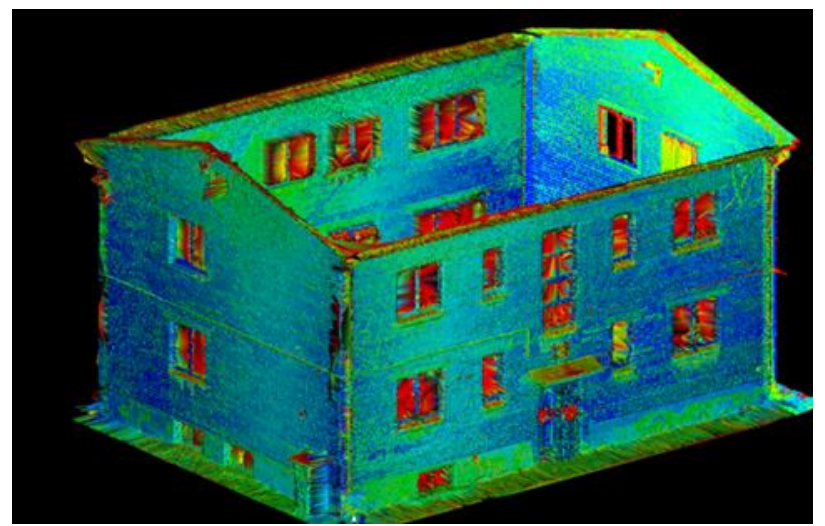

Fig. 3. Surface model of a two-story apartment building visualized with Leica Cyclone software

For the purpose of visualization of the point clouds and collaboration between all stakeholders a free online viewing and markup tool Faro WebShare was. Data were shared in the cloud to make them available for inspection with conventional browser. Data may be also embedded into a client's website. This allows all participants to observe the original site in real situation later at any time during any stage of the collaboration process. Any further measurements between elements may be performed directly in the visualized point cloud media by means of web browser without installing any additional software. As an alternative online data viewer a Leica Truview browser may be used.

3D point clouds are available in a variety of industry standard formats including *.las, *.pts, *.ptx, *.pcg, *.pod, *.txt etc. which are registered and optionally coordinated to fit all the most popular practically used systems.

\subsection{Data post-processing}

The post-processing using software may be performed in two different ways as follows:

- With specially developed or dedicated software, or

- $\quad$ Importing directly into existing specialized end user software.

Specially developed software that is dedicated for particular tasks can process large amounts of scanning data, and create different results, including support for a CAD model, mesh-model, cross-section, etc. The results later can be exported into other systems, such as CAD, GIS, BIM or other user-familiar systems for different applications.

A surface modeling of the building included the following steps:

- Creation of mesh,

- Creation of path surface (B-spline sur-face),

- Creation of SmartSurface.

The most popular surface generation meth-od is a triangulation. For this purpose different type of software may be used: post-processing (Farro Scene, Leica Cyclone, etc.), CAD soft-ware (Autodesk, Bentley, etc.) or specialized for surface generation (MeshLab, Geomagic Studio, 3DReshaper, etc.

Triangulated surface mesh is quite complex and problematic for the further use in most popular CAD formats. The mesh model size for the surfaces of all walls exceeded $100 \mathrm{MB}$. It was decided to use additional software and convert the mesh surface model into patch model (Geomagic Studio) or SmartSurface model (Bentley). The software uses other surface interpolation algorithms and after trans-formation the obtained surface models are much simpler and smaller, and easier for analyzing the deviations from the exact geometry. 
The existing information captured as point cloud data usually has a higher accuracy than the existing pre-construction drawings in blue-print format. Therefore the created 3D surface meshes can be used to create deliverables in the form of 2D drawings which may be imported into wide range of CAD or BIM applications. 3D model deliverables should be created using standard formats, such as *.ifc, or common exchange formats like *.dxf to allow clients to retain as much data intelligence as possible. However, mesh models capture only the geometric properties of the building and are not suitable for direct use as BIM models.

Traditional 2D deliverables (plans, sections, elevations, details, etc.) can be generated directly from the point cloud data by taking virtual sections through the point cloud and generating drawings in conventional *.dwg or *.dxf format. The surface accuracy of point clouds is high, making this a superior method for reproducing existing conditions in comparison to manual measurements.

When the end deliverable of the visualization of the scanned environment is animated in fly-through mode, a HD quality movie files are easy available along with planned design features added. Using high definition point clouds the detailed 2D elevation drawings may be extracted that are delivered in *.dwg or *.dxf format or alternatively scaled ortho photograph elevations of the cleaned scan data. From the 3D laser scan data fully rendered visualizations can be pictured from any viewpoint to provide detailed graphics of existing structures or environments and optionally incorporated design elements. The results of thermographic measurement may be superimposed on the visualizations of the scanned building [15].

\subsection{The capture of BIM model}

The building elements in a BIM model which are of particular interest in the present study are those which exchange energy with the environment, namely, walls, slabs, foundation, roofs, windows, doors, and the soil of the terrain which is in contact with building foundation elements. None of the deliverables available in the 3D scan workflow described above provide a model in a BIM compatible format which is required for further energy analysis.

Manual, semi-automatic or automatic processes for BIM model creation are distinguished when capturing the external and/or internal elements of the building [15]. Some third party application designers have developed tools which are usable in Revit, ArchiCAD or AECOsim, and etc. software for automation of conversion process. However, according to [16] in practice a 3D model generation from point cloud data and setup for energy analysis so far is a time-consuming and labor intensive manual process subjected to numerous errors.

BIM models in this study were produced directly from the point cloud data. Autodesk ReCap freeware was used to cope with differ-ent scan data file formats (*.rcp, *.rcs, *.e57) to share the data with Revit or ArchiCAD software if the scan data did not meet the requirements. The imported point cloud was adjusted to the the zero level of the ground floor location and the slab height was referenced with respect to the window sill and corresponding room height dimensions which were retrieved and documented during the on-site visit.

An approach of manual BIM model production proved to be the most efficient and fastest workflow as compared to conventional on-site measurements. This method also minimizes efforts and time spent and cost of post-work when finding and fixing errors after automatic BIM model generation process [17-18].

Automatic process of BIM model creation has limited success for old buildings also due to some other practical issues. One is related to the foundation settlement which causes 
serious problems for the design of prefabricated insulation panels. The foundation settlement of the building in present study was almost $13 \mathrm{~cm}$ on the $10 \mathrm{~m}$ long base (Figure 4). The window openings are neither rectangular nor orthogonal to the vertical and horizontal directions.

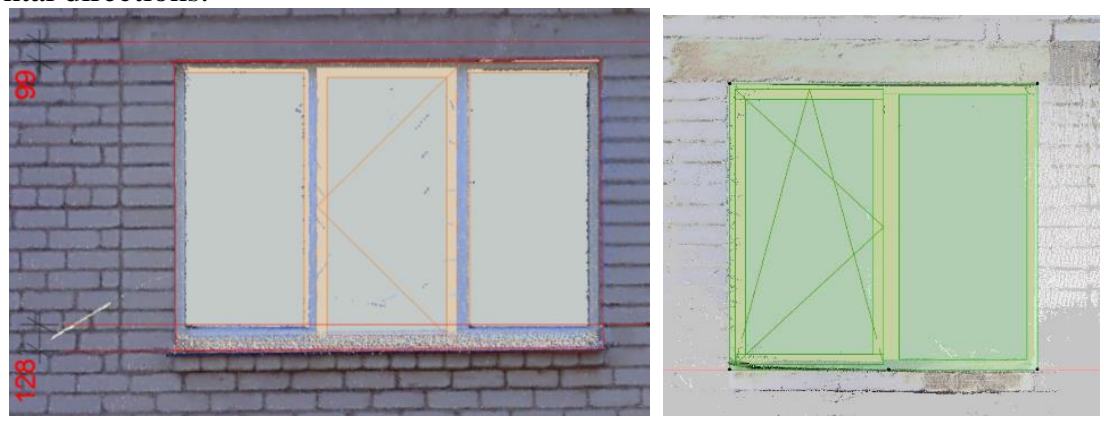

Fig. 4. The height difference between left and the most right window on the North façade

Another factor preventing automatic recognition is that in practice the external walls very often are neither planar nor strictly vertical.

Local cross sections in the point cloud were identified at different story heights and used for existing external wall tracing. To create a medium fidelity BIM model the interior walls were also included in the model. Since the rooms of the residential building are relatively small with a lot of indoor occlusions (furniture) the scan process is not effective. Instead the existing inventory plan drawings were digitally referenced to the BIM model and internal walls were manually traced over.

Manually created as-is BIM model provides information at the element level (walls, slabs, roofs, soil, windows and doors). Elements can be summarized by a small number of parameters therefore BIM model has the capability to supplement the 3D data with additional intelligent and semantic attributes before sharing it with other stakeholders for the use in external analysis applications. Since the BIM software provides easy and intuitive interface for visual representation of geometric model and element manipulation, it is very convenient for the definition of physical parameters of the elements considered during analysis either directly in the original media or in the applications of the third party developers.

The prepared BIM model of the building (Figure 9) consists from parametric building elements with customizable semantic parameters. Model can be exported to different analysis applications in different formats. Estimation of final energy consumption strongly depends on input data quality [19 and 20], 3D scanning allows precise model import in major formats which are used by designer energy auditors and construction companies.

\section{Conclusions}

A laser scanning process is the fastest method of 3D data acquisition for the existing buildings. The selection of the scanner type and scanning setup depends on the architecture or geometric complexity of the building elements.

The accuracy of the points in the point cloud and average spacing between points in the processed point cloud is within $3 \mathrm{~mm}$ range. The accuracy of the surface model automatically generated from the point cloud with standard algorithms is within $5-10 \mathrm{~mm}$.

The accuracy of a manually traced BIM model of the building highly depends on the accuracy of point cloud and the experience and the skills of the modeler. The tested 
building example is very simple therefore in the case of realistic and more complex buildings more precise scan data might be required.

The created BIM model for preliminary energy analysis may be used also for the second goal in this study that was the collection of geometric information for the design of prefabricated wood panel. The wood panel de-signers use very specific software and prefer conventional AutoCAD drawings rather than 3D model in any form. 3D models may serve only as supplemental informational for the project. The set of plan view drawings and drawings of all the façades along with characteristic sections in *.dwg format will carry the necessary geometry information for the panel design.

This study was supported by European Regional Development Fund project.

\section{References}

1. P. Op't Veld. MORE-CONNECT: Development and Advanced Prefabrication of Innovative, Multifunctional Building Envelope Elements for Modular Retrofitting and Smart Connections. Energy Procedia, 78:1057-1062. (2015)

2. W. Böhler, G. Heinz and A. Marbs. The Potential of Non-Contact Close Range Laser Scanners for Cultural Heritage Recording. In: Proc. of XVIII Int. Symp. of CIPA 2001. Surveying and Documentation of Historic Buildings - Monuments - Sites. Traditional and Modern Methods. Potsdam, Germany, September 18-21. -p. 430-436.( 2001)

3. F. Remondino and S. Campana. 3D Recording and Modelling in Archaeology and Cultural Heritage: Theory and Best Practices. Archaeopress: Oxford. -171 p. (2014)

4. D. Gleason. Laser Scanning for an Integrated BIM. In: Lake Constance 5D Conference, Constance, Germany, October 28-29 -8 p. (2013)

5. C. Thomson and J. Boehn. Automatic Geometry Generation from Point Clouds for BIM. Remote Sens., 7:11753-11775.( 2015)

6. Y.K. Juan, P. Gao and J. Wang. A hybrid decision support system for sustainable office building renovation and energy performance improvement. Energy and Buildings, 2010, 42 (3):290-297.

7. M. Aldanondo, A. Barco-Santa, E.Vareilles, M. Falcon, P. Gaborit and L. Zhang. Towards a BIM Approach for a High Performance Renovation of Apartment Buildings. In: Product Lifecycle Management for a Global Market. Revised Selected Papers of the 11th IFIP WG 5.1 Int. Conf., PLM 2014, Yokohama, Japan, July 7-9, 2014. -p. 21-30.

8. C. Eastman, P. Teicholz, R.Sacks and K. Liston. BIM Handbook. A Guide to Building Information Modeling for Owners, Managers, Designers, Engineers, and Contractors. John Wiley \& Sons, Inc. 2nd ed., 2011. -648 p.

9. K.P. Kim and K. Park. BIM feasibility study for housing refurbishment projects in the UK. Organization, Technology \& Management in Construction, 2013, 5(Special):756774.

10. R. Volk, J. Stengel and F. Schultmann. Building Information Modeling (BIM) for existing buildings - Literature review and future needs. Automation in Construction, 2014, 38:109-127.

11. P. Tang, D. Huber, B. Akinci, R. Lipman and A. Lytle. Automatic reconstruction of asbuilt building information models from laser-scanned point clouds: A review of related techniques. Automation in Construction, 2010, 19: 829-843.

12. E. Rojas, C. Dossick, J. Schaufelberger. Developing Best Practices for Capturing AsBuilt Building Information Models (BIM) for Existing Facilities. In: Report of Construction Engineering Resaearch Laboratory ERDC/CERL CR-10-2, 2010. -91p. 
13. Level of Development Specification for Building Information Models. BIM Forum, Version. 2015. -195 p.

14. Y. Arayici. Towards Building Information Modelling For Existing Structures. Structural Survey, 2008, 26 (3): 210-222.

15. C. Wang, Y.K. Cho. Performance Evaluation of Automatically Generated BIM from Laser Scanner Data for Sustainability Analyses. Procedia Engineering, 2015, 118: 918 925.

16. P. Tang, D. Huber, B. Akinci, R. Lipman, A. Lytle. Automatic reconstruction of as-built building information models from laser-scanned point clouds: A review of related techniques. Automation in Construction, 2010, 19:929-843.

17. Y.K. Cho, Y. Ham, M. Golpavar-Fard. 3D as-is building energy modeling and diagnostics: A review of the state-of-the-art. Adv. Eng. Informatics, 2015, 29:184-195.

18. X. Xiong, A. Adan, B. Akinci, D. Huber. Automatic creation of semantically rich 3D building models from laser scanner data. Automation in Construction, 2013, 31:325337.

19. Z. Verbai. A Lakatos A. \& F Kalmár. Prediction of energy demand for heating of residential buildings using variable degree day. Energy. 2014. No. 76. pp. 780-787. doi:10.1016/j.energy.2014.08.075

20. K Kuusk, \& T Kalamees. Retrofit cost-effectiveness: Estonian apartment buildings. Building Research and Information. No. 44(8). 2016. Pp. 920-934. doi:10.1080/09613218.2016.110311.

21. M Petritchenko, A Subbotina, F Khairutdinova, V Reich, D Nemova, Ya Olshevskiy, V Sergeev. Effect of rustication joints on air mode in ventilated facade (2017) Magazine of Civil Engineering, 73 (5), pp. 40-48. DOI: 10.18720/MCE.73.4 